\title{
Advances in the therapy of gastric cancer
}

\author{
JoHn S. Macdonald \\ Saint Vincents Comprehensive Cancer Center, 325 West 15th Street, New York, New York 10011, USA
}

\begin{abstract}
Many issues remain unclear in the management of gastric cancer. Randomized trials have failed to show the superiority of D2 over D1 dissection, and comparisons between countries showing higher survival rates following more extensive surgery may be influenced at least in part by the fact that D1 dissection underestimates disease stage in many patients. No studies have yet shown a benefit from adjuvant chemotherapy. However, the Southwest Oncology Group (SWOG) 9008 trial provides convincing evidence that a regimen of postoperative 5-fluorouracil (5-FU)-based chemoradiotherapy improves disease-free and overall survival when compared with observation alone. In the chemotherapy of advanced disease, use of the epirubicin plus cisplatin plus 5-FU (ECF) regimen leads to significantly longer median survival than 5-FU plus adriamycin plus methotrexate-C (FAMTX), but the rate of complete response remains low and the effect on longterm survival minimal. There are indications that neoadjuvant chemotherapy may increase the resectability of tumors and reduce risk of postoperative recurrence. Substantial improvements in outcome are likely to depend on the integration into multimodality strategies of novel, molecularly targeted agents in all stages of gastric cancer treatment.
\end{abstract}

Key words Gastric cancer · Adjuvant chemotherapy · Radiation

\section{Introduction}

In the United States, there are almost 22000 new cases of gastric cancer annually, and 13500 deaths from the disease. Worldwide, 800000 people are diagnosed each year as suffering from gastric cancer. In the United

Offprint requests to: J.S. Macdonald

John S. Macdonald has received honoraria and medical research grants from Aventis Pharmaceuticals Inc.

Received: April 1, 2002; Accepted: June 18, 2002
States, adenocarcinoma of the stomach was the most common cause of cancer-related deaths in 1900 [1] and fell dramatically in the latter half of the century [1]. The gastric cancer incidence rate of roughly 35 per 100000 in 1930 fell to approximately 3 per 100000 in the United States in the 1970s [2]. There has been no adequate explanation for this change. It is of interest that the fall in gastric cancer has occurred in the "endemic" or intestinal form [3] of the disease that is usually associated with preexisting intestinal metaplasia. This is the form of gastric cancer seen in high-incidence countries and appears to result from a combination of achlorhydria, migration of small intestinal epithelium into the stomach resulting in intestinal metaplasia, and superinfection with Helicobacter pylori [4]. This combination over time results in chronic gastritis with dysplastic changes and finally the development of frank adenocarcinoma of the stomach. This form of gastric cancer is the histopathologic type of cancer that has decreased significantly in incidence in the United States over the last 70 years.

Although the endemic form of stomach cancer is decreasing, there is another type of gastroesophageal cancer that has increased in incidence. These are tumors that do not occur on the background of intestinal metaplasia but develop in the gastroesophageal junction and distal esophagus, and are cancers associated with Barrett's metaplasia developing from chronic esophageal reflux disease (GERD) [5,6]. These cancers occur most commonly in middle-aged Caucasian males and most frequently involve lower esophagus plus gastroesophageal junction. It becomes difficult to determine whether these are originating in the stomach or distal esophagus. In many clinical trials and in clinical-care guidelines, proximal gastric and distal esophageal cancers are treated in the same manner. Of note, it is likely that the etiologic factors associated with the endemic form of gastric cancer and the distal esophageal cardiac junction epithelium cancers are mutually exclusive. 
Achlorhydria and $H$. pylori infection $[4,6]$ would be protective against the development of Barrett's esophagus-associated cancers $[1,6]$, which are associated with acid reflux into the esophagus, and, likewise, the high acid content in the stomach is incompatible with achlorhydria, $H$. pylori infection, and subsequent development of the endemic form of adenocarcinoma.

Despite secular trends showing reduced incidence of the disease over many decades, gastric cancer remains a widespread and intractable public health problem, which deserves the urgent and sustained attention of the clinical and research community.

\section{Surgery}

The primary curative treatment of gastric carcinoma and distal esophageal cancer is surgical resection [7-9]; however, there is no global consensus on how extensive the surgery should be. In Japan, the standard of care is the D2 dissection, in which the N2 nodes are taken en-bloc with the cancer. In the United States, the recommended procedure is less radical, with the removal of N1 nodes only.

In stomach cancer potentially resectable for cure (stage 0-IV M0), the surgical aim should be to perform a tumor resection entailing at least a partial gastrectomy with an en-bloc dissection of lymphatic tissue. For at least 20 years $[7,8]$, there has been an international debate regarding the most appropriate surgical procedures to use in cases of potentially curable gastric carcinoma. The point at issue is whether an extensive lymph node dissection improves survival. If a surgeon dissects in an en-bloc fashion, all of the cancer plus the N2 lymph nodes, a D2 dissection has been performed. The D2 dissection as the norm for gastric cancer surgery developed from surgical practice in Japan. Japanese surgeons [8] for a number of years have reported superior results with this type of surgical resection in gastric cancer.

At least four controlled studies have randomized patients to D1 versus D2 dissection [9-12]. These include the relatively small phase III comparisons of D1 and D2 dissections that have been completed in South Africa [10] and Hong Kong [11]. These studies were underpowered (fewer than 30 patients per arm) and showed no survival benefit for D2 resection. A much larger phase III study [9] with 996 cases also evaluated D2 vs D1 dissection. This study also demonstrated that the D2 dissection did not appear overall to improve survival and was associated with a higher morbidity. This study is also of interest for an earlier evaluation of the effect of extended dissection on staging accuracy. Importantly, there is evidence from the Dutch study that the extent of dissection does indeed influence disease stag- ing. A report by Bunt et al. [12] in 1995 demonstrated that patients undergoing D2 dissections had a significantly more accurate surgical pathological staging than patients undergoing D1 dissections. Of 48 patients classified as stage II on D1 criteria, 18 (38\%) were reclassified as stage IIIA on information from the more radical dissection. Of 49 patients judged to be stage IIIA on D1 data, $21 \%$ became stage IIIB and 1 stage IV with the additional D2 information (a 45\% shift in stage). Of 24 initially IIIB patients, 18 became stage IV when N2 nodes were taken into account ( $75 \%$ shift). The use of a D1 dissection appeared to understage patients compared with a D2 dissection approximately $50 \%$ of the time. In evaluating the effect of postoperative systemic therapy in gastric cancer it is important to consider that resections that use less than $\mathrm{D} 2$ dissections result in a significant risk of understaging.

Comparison of survival experience between the United States and Japan shows marked differences. In the United States, patients with disease classified as stage I who had surgery over the period 1982-1987 had a $50 \%$ 5-year survival rate. Of stage I patients operated on in Japan between 1971 and 1985, 91\% lived to 5 years. Similar discrepancies exist for more advanced disease: for stage II patients, the survival rates in the United States were 29\% [13,14] and in Japan 72\%; for stage III patients, the corresponding figures were $13 \%$ and $44 \%$; and for stage IV disease, $3 \%$ and $9 \%$. There are competing explanations for these marked differences in outcome. They may arise because radical surgery is indeed more likely to be curative. On the other hand, it may be that by providing information on a larger number of nodes, D2 dissection allows the more accurate staging of patients, leading to the assignment of greater numbers to categories of more advanced disease.

The Western patients, despite an RO resection, remain at substantial risk for developing local and systemic cancer. Thus, these patients would be excellent candidates for post-resection adjuvant therapy aimed at destroying microscopic metastatic cancer.

\section{Adjuvant therapy}

As in other types of tumor, the purpose of adjuvant therapy in gastric cancer is to eradicate microscopic metastastic disease, so reducing the rate of relapse and increasing the likelihood of cure.

Over several decades, individual studies of adjuvant chemotherapy in gastric cancer and metaanalyses based on pooled data from ten published randomized studies have failed to show any significant benefit arising from the use of adjuvant regimens [14]. In the United States [13], a clinical trial testing FAM (5-FU, adriamycin, 
mitomycin-C) chemotherapy in a cooperative group (Southwest Oncology Group; SWOG) also did not demonstrate any benefit for adjuvant chemotherapy. In this study [13], 191 patients were randomized between 1 year of FAM following surgery or surgery alone. There was absolutely no benefit for chemotherapy, with the survival curves being overlapping. The overall survival at 5 years demonstrated in the study was approximately $35 \%$ for surgery or surgery and FAM chemotherapy.

One of the important findings in gastric cancer over the last 15 years has been that, in patients with known residual disease, the combination of radiation therapy plus fluorinated pyrimidine (5-FU) used as a radiation sensitizer could result in the complete control (apparent cure) of small amounts of residual or recurrent stomach cancer [15]. This use of combined modality radiation and chemotherapy has also been demonstrated to be efficacious in esophageal cancer [16] and has resulted, in that disease, in a prolonged disease-free survival of patients without the need for surgical resection. Because of the demonstrated benefit for combined radiation and fluorinated pyrimidine in patients with known residual gastric and esophageal carcinoma, a United States Intergroup study (SWOG 9008) was initiated in the early 1991 to test whether the combination of 5-FU/leucovorin plus radiation therapy after surgical resection would be of value to patients with resected gastric carcinoma. This study enrolled 603 patients in 7 years of accrual and was reported in the spring of 2000 [17].

The SWOG 9008/Intergroup [17] trial is a landmark study in the adjuvant treatment of gastric cancer. In this trial, good performance status patients with stage IB-1V M0 gastric adenocarcinoma who had had en-bloc resection and negative surgical margins were randomized after their operation to either an observation arm or to adjuvant chemoradiotherapy. This consisted of 5-FU/ leucovorin followed by $4500 \mathrm{cGy}$ radiation accompanied by 5-FU/LV and followed by a further two cycles of the same chemotherapy regimen (Fig. 1). Of the 603 patients registered, all but 47 were judged eligible (ineligibility arising in the majority of cases from positive surgical margins).
Among the 273 patients assessable for toxicity, grade 3 or 4 hematological events occurred in $54 \%$. Gastrointestinal toxicity was seen in 33\%, a flu-like syndrome in $9 \%$, and infection in $6 \%$. Grade $3 / 4$ neurological and cardiac toxicity was reported in $4 \%$. Three deaths occurred: there was one case of myocardial infarction, which may not have been treatment-related, and one case each of pulmonary fibrosis and neutropenic sepsis, which were clearly treatment-related.

Patients with resected gastric cancer may be undernourished, and in otherwise poor condition. Combined modality therapy including upper abdominal radiation must be approached with caution in this group. Given careful planning, the experience of the SWOG trial suggests that chemoradiotherapy is reasonably well tolerated.

In considering the survival outcome of the trial, it should be noted that $54 \%$ of patients included had a less than D1 dissection (involving incomplete removal of N1 nodes), while $36 \%$ had a D1 dissection. In only $10 \%$ was the $\mathrm{D} 2$ procedure used. Based on data noted above, it is therefore likely that $60 \%-70 \%$ of patients had residual cancer in nodes not removed during surgery.

The outcome of the trial was exceptionally positive. Compared with patients in the observation-only arm, those treated with chemoradiotherapy experienced significantly longer progression-free survival (median, 30 vs 19 months; $P=0.0001)$ and significantly longer overall survival ( 35 vs 28 months; $P=0.01$ ). The latter data are based on a median follow-up of 4.9 years.

Of the 177 patients with recurrence in the observation group, $29 \%$ had recurrences that were local (i.e., in the stomach or operated area), $72 \%$ had regional recurrences (including abdominal carcinomatosis and liver metastases), and $18 \%$ had distant (i.e., extraabdominal) failure. Among the 120 chemoradiotherapy-treated patients who developed a recurrence, $19 \%$ had local recurrence, $65 \%$ regional recurrence, and $33 \%$ a distant recurrence.

The improved survival following chemoradiotherapy in resected gastric cancer is achieved with acceptable toxicity, although careful attention must be paid to

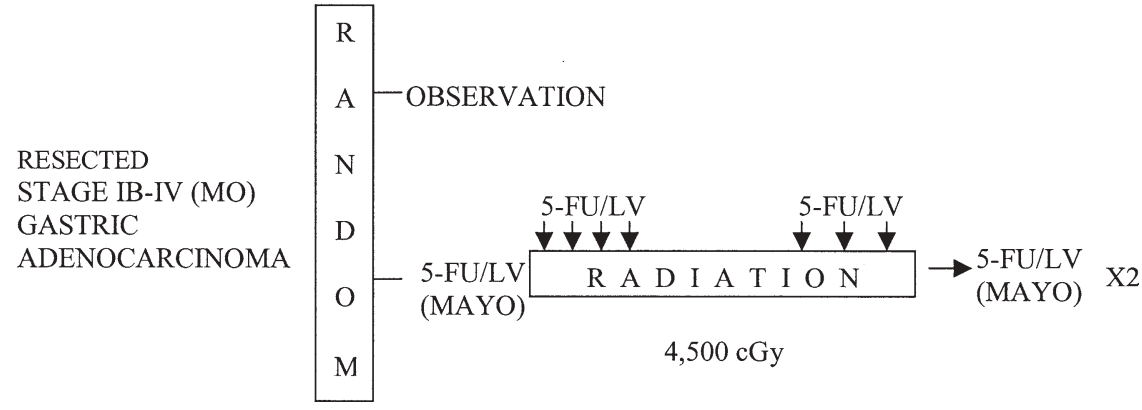

Fig. 1. Southwest Oncology Group (SWOG) 9008/INT 0116: study schema [17]. 5-FU, 5-fluorouracil; $L V$, leucovorin 
radiation planning and delivery so that the heart, liver, and kidneys are spared. In high-risk patients, postoperative chemoradiotherapy should be considered the standard of care.

It should be noted that the SWOG study discussed above was designed in 1990 and used a cytotoxic regimen current at that time. With the advent of newer agents, such as irinotecan, docetaxel, and agents with small molecules, it may now be possible to achieve similar benefit using adjuvant chemotherapy alone.

\section{Chemotherapy}

A variety of combination chemotherapy regimens has been widely used in a palliative management of patients with gastric cancer [18-23]. None of these regimens results in cure of adenocarcinoma of the stomach when the cancer has already disseminated and, although some of them are associated with complete response (CR) rates as high as $15 \%$, these CRs are not durable.

The FAM regimen (consisting of $5-\mathrm{FU} 600 \mathrm{mg} / \mathrm{m}^{2}$ given in weeks $1,2,5,6$, and 9 , together with doxorubicin $30 \mathrm{mg} / \mathrm{m}^{2}$ every 3 weeks, and mitomycin-C $10 \mathrm{mg} / \mathrm{m}^{2}$ in weeks 1 and 9) played the important role of demonstrating that the cancer is chemosensitive. Indeed, the achievements of the FAM regimen should not be underestimated. In a small meta-analysis in 1984 of eight clinical trials, including a total of 302 cases, the response rate (RR) was $35 \%[13,24]$. The median survival time was 6.2 months, which is not dissimilar from that achieved by many regimens over the past two decades. However, the rate of CR was only $2 \%$, and evidence from other cancer, such as ovarian cancer, suggests that only patients who have a very substantial regression of cancer are likely eventually to show survival benefit.

A second regimen of interest is ECF, consisting of epirubicin $50 \mathrm{mg} / \mathrm{m}^{2}$ and cisplatin $60 \mathrm{mg} / \mathrm{m}^{2}$ every 3 weeks plus $5-\mathrm{FU} 200 \mathrm{mg} / \mathrm{m}^{2}$ per day by continuous infusion. Webb et al. [21] compared ECF against 5-FU, adriamycin and methotrexate (FAMTX) in a randomized study of 219 patients with advanced and locally advanced gastroesophageal adenocarcinoma [21]. ECF proved significantly superior to FAMTX, achieving an overall RR of $45 \%$ vs $21 \%(P=0.0002)$. However, the responses were not confirmed. The rates of CR were $6 \%$ and $2 \%$. Median survival was significantly longer in the ECF group. However, use of ECF was associated with little improvement in the proportion of patients living beyond $1-2$ years.

Among more recent developments, the use of a weekly low-dose irinotecan and cisplatin combination has seemed promising. In the study by Ilson et al. [25], $65 \mathrm{mg} / \mathrm{m}^{2}$ weekly irinotecan was given with $30 \mathrm{mg} / \mathrm{m}^{2}$ cisplatin to patients with esophageal cancer (the major- ity of whom had distal tumors). The RR among 23 patients with adenocarcinomas was $57 \%$ and that among 12 patients with squamous cell tumors, $66 \%$. Grade 4 neutropenia occurred in $9 \%$ of patients. Grade 3 diarrhea was reported in $11 \%$, suggesting that this irinotecan-associated toxicity is minimized by weekly low-dose administration.

Docetaxel is another relatively recent agent extensively studied in gastric cancer, alone and in combination. (The controlled TAX 325 trial of docetaxel plus cisplatin vs docetaxel plus cisplatin and 5-FU, for example, is described in detail elsewhere in this volume [26]) Recent attention has been drawn to the role of dose intensity. Relevant to this is the work of Chun et al. [27], who studied a weekly docetaxel-based regimen in 15 medically frail gastric cancer patients aged 70 years and older. Weekly docetaxel was given at $25 \mathrm{mg} / \mathrm{m}^{2}$ together with continuous infusion $5-\mathrm{FU} 150 \mathrm{mg} / \mathrm{m}^{2}$ for 14 days in each 3 -week cycle. One of 15 patients $(7 \%)$ achieved a complete response and a further 11 a partial response $(73 \%)$. Toxicity was minimal.

Of studies using a more conventional schedule, the German work on docetaxel $75 \mathrm{mg} / \mathrm{m}^{2}$ plus cisplatin $75 \mathrm{mg} / \mathrm{m}^{2}$ every 3 weeks is encouraging [28]. Among 85 evaluable patients, the CR rate was $8 \%$ and the partial response rate $28 \%$, giving an overall RR of $37 \%$. Median survival was 10 months. Among 96 patients evaluable for toxicity, who received a median of 5.5 cycles, the rate of grade 4 myelotoxicity was $4 \%$. No grade 4 gastro-intestinal or neurological toxicity or alopecia was seen.

\section{Neoadjuvant therapy}

The next area of investigation for the use of systemic therapy in gastric cancer will be to test in a critical fashion the use of preoperative or neoadjuvant chemotherapy. Neoadjuvant treatment before attempts at surgical resection, chemotherapy, and/or radiation therapy of gastric cancer has not been tested in controlled randomized trials. However, the results of phase II studies have been published. Clinical investigators of Memorial Sloan Kettering in New York [29] and the University of Southern California [30] have reported results of this therapeutic approach. Both of these groups have used systemic preoperative chemotherapy along with a post-resection intraperitoneal chemotherapy strategy designed to prevent relapse in the peritoneal cavity. Intraperitoneal chemotherapy is a rational approach, because the peritoneal cavity is a common site of recurrence after gastric resection.

An example of such aggressive neoadjuvant therapy is the 1997 report by Cookes et al. at the University of Southern California [30]; in this study, 59 patients 
received combined modality therapy consisting of continuous infusion 5-FU with weekly leucovorin and cisplatin. This was followed by surgical resection of the primary gastric tumor. Postoperatively, resected patients received two cycles of intraperitoneal 5-fluoro-2deoxyuridine and intraperitoneal cisplatin. This study demonstrated that 56 of $59(95 \%)$ patients receiving neoadjuvant therapy were able to undergo surgical resection. Forty patients $(71 \%)$ underwent resections with curative intent. Only 9 of $40(23 \%)$ patients who had curative resection developed recurrent carcinoma. The median time in follow-up now exceeds 45 months. The observed relapse rate in well-designed prospective studies after gastric cancer resection is in the range of $60 \%-70 \%[13,14]$. The $23 \%$ relapse rate in the University of Southern California study with a median followup of almost 4 years is an impressive phase II result. What must be done in the future is to subject neoadjuvant therapeutic approaches such as that described by Cookes et al. [30] to evaluation in prospectively randomized clinical trials. A neoadjuvant approach will be one of the strategies tested in the next national early gastric cancer intergroup study.

\section{Discussion}

The future is likely to see the development of strategies which combine nonspecific cytotoxic agents with drugs aimed at molecules such as the epidermal growth factor receptor (EGFR), a target of considerable relevance in gastric cancer. Immunohistochemistry carried out during the SWOG 9008 study showed overexpression of EGFR in $75 \%$ of cancers. Cancers from this trial are also being studied to determine whether those that were fundamentally anti-apoptotic in character (as indicated, for example, by overexpression of bcl-2) responded less well to cytotoxic therapy than those with pro-apoptotic characteritics. Factors related to the triggering of apoptosis, such as the presence of wild-type p53, also suggest a possible role for gene therapy.

The large quantities of cancer available from resected patients offer the possibility of using biology to define the susceptibility of individual cancers to the range of new approaches, such as EGFR, angiogenesis, or ras inhibitors.

Based on the SWOG 9008 findings, some form of adjuvant chemoradiotherapy is clearly appropriate for many patients. This is likely to be based on more recent cytotoxic agents, but will hopefully incorporate molecularly targeted drugs selected on the basis of individual tumor biology.

In this respect, oncology may be on the cusp of a therapeutic revolution similar to that which transformed the treatment of infectious disease. In the early 1900s, therapy relied on nonspecific and highly toxic agents such as cyanides, arsenicals, and heavy metals. These were replaced in mid-century by penicillin, an agent targeted very specifically at the bacterial cell wall. In the early years of the new century, cancer medicine may be undergoing a similar paradigm shift.

In the meanwhile, use of conventional agents can be more finely tuned to optimizing the balance between efficacy and toxicity. There are groups of patients who are elderly or of poor performance status, for example, who appear to derive a high rate of clinical response with weekly, low-dose administration of agents such as irinotecan or docetaxel in combination with cisplatin or 5-FU. If these early findings can be confirmed, such regimens would seem to have considerable potential for the effective palliation of advanced disease.

\section{References}

1. Gunderson LL, Donohue JH, Burch PA. Stomach. In: Abeloff $\mathrm{MD}$, Armitage JO, Lichter AS, Niederhuber JE, editors. Clinical oncology. New York, NY: Churchill Livingstone; 1995. p 1209-41.

2. Greenlee RT, Murray T, Bolden S, Wingo PA. Cancer statistics 2000. CA Cancer J Clin 2000;50:7-33.

3. Boring $\mathrm{H}$. Epidemiological research in stomach cancer: progress over the last 10 years. J Cancer Res Clin Oncol 1991;117:13343.

4. Parsonnet J, Friedman GD, Vandersteen DP, Chang Y, Vogelman JH, Orentreich N, et al. Helicobacter pylori infection and the risk of gastric carcinoma. N Engl J Med 1991;325:1127-31.

5. Wu-Williams AH, Yu MC, Mack TM. Life-style, workplace, and stomach cancer by subsite in young men of Los Angeles county. Cancer Res 1990;50:2569-76.

6. Macdonald JS, Hill MC, Roberts IM. Gastric cancer: epidemiology, pathology, detection and staging. In: Ahlgren JD, Macdonald JS, editors. Gastrointestinal oncology. Philadelphia: J.B. Lippincott; 1992. p. 151-8.

7. Vezerdis MP, Wanebo HJ. Gastric cancer: surgical approach. In: Ahlgren JD, Macdonald JS, editors. Gastrointestinal oncology. Philadelphia: J.B. Lippincott; 1992. p. 159-70.

8. Kodama Y, Sugimachi K, Soejima K, Matsusaka T, Inokuchi K. Evaluation of extensive lymph node dissection for carcinoma of the stomach. World J Surg 1981;5:241-8.

9. Bonenkamp JJ, Hermans J, Sasako M, van de Velde CJH, Welvaart K, Songun I, et al. Extended lymph node dissection for gastric cancer. N Engl J Med 1999;340:908-14.

10. Dent DM, Madden MV, Price SK. Randomized comparison of $R_{1}$ and $\mathrm{R}_{2}$ gastrectomy for gastric carcinoma. Br J Surg 1988;75:1102.

11. Robertson CS, Chung SC, Woods SD, Griffin SM, Raimes SA, Lan JT, Li AK. A prospective randomized trial comparing R1 subtotal gastrectomy with R3 total gastrectomy for antral cancer. Ann Surg 1994;220:176-82.

12. Bunt AMG, Hermans J, Smit VT, van de Velde CJ, Fleuren GJ, Bruijn JA. Surgical/pathologic-stage migration confounds comparisons of gastric cancer survival rates between Japan and Western countries. J Clin Oncol 1995;13:19-25.

13. Macdonald JS, Fleming TR, Peterson RF, Berenberg JL, McClure S, Chapman RA, et al. Adjuvant chemotherapy with 5$\mathrm{FU}$, adriamycin, and mitomycin-C (FAM) versus surgery alone for patients with locally advanced gastric adenocarcinoma: a Southwest Oncology Group Study. Ann Surg Oncol 1995;2:48894. 
14. Hermans J, Bonenkamp JJ, Boon MC, Bunt AM, Ohyama S, Sasako M, et al. Adjuvant therapy after curative resection for gastric cancer: meta-analysis of randomized trials. J Clin Oncol 1993;11:1441-47.

15. Gastrointestinal Tumor Study Group. A comparison of combination chemotherapy and combined modality therapy for locally advanced gastric carcinoma. Cancer 1982;49:1771-7.

16. Herskovic A, Martz K, Al-Sarraf M, Leichman L, Brindle J, Vaitkevicius V, et al. Combined chemotherapy and radiotherapy compared to radiotherapy alone in patients with cancer of the esophagus. N Engl J Med 1992;326:1593-8.

17. Macdonald JS, Smalley S, Benedetti J, Estes N, Haller D, Ajani J, et al. Postoperative combined radiation and chemotherapy improves disease-free survival and overall survival in resected adenocarcinoma of the stomach and G.E. junction. Results of Intergroup Study INT-0116 (SWOG 9008) (abstract). Proc Am Soc Clin Oncol 2000;19:1.

18. Wils J, Bleiberg H, Dalesio O, Blijham G, Mulder N, Planting A, et al. An EORTC gastrointestinal evaluation of the combination of sequential methotrexate, and 5-fluorouracil combined with adriamycin, in advanced measurable gastric cancer. J Clin Oncol 1986;4:1799-1803.

19. Kelsen D, Atiq OT, Saltz L, Niedzwiecki D, Ginn D, Chapman D, et al. FAMTX versus etoposide, doxorubicin, and cisplatin: a random assignment trial in gastric cancer. J Clin Oncol 1992; 10:541-8.

20. Wils JA, Klein HO, Wagener DJT, Bleiberg H, Reis H, Korsten $\mathrm{F}$, et al. Sequential high-dose methotrexate and fluorouracil combined with doxorubicin: a step ahead in the treatment of advanced gastric cancer: a trial of the European Organization for Research and Treatment of Cancer Gastrointestinal Tract Cooperative Group. J Clin Oncol 1991;9:827-31.

21. Webb A, Cunningham D, Scarffe JH, Hazpez P, Norman A, Joffe $\mathrm{JK}$, et al. Randomized trial comparing epirubicin, cisplatin and fluorouracil versus fluorouracil, doxorubicin, and methotrexate in advanced esophagogastric cancer. J Clin Oncol 1997;15:261-7.

22. Cascinu S, Labianca R, Allesandroni P, Marcellini M, Silva RR, Pancera G, et al. Intensive weekly chemotherapy for advanced gastric cancer using fluorouracil, cisplatin, epi-doxorubicin, 6Sleucovorin, glutathione, and filgrastim: a report from the Italian Group for the Study of Digestive Tract Cancer. J Clin Oncol 1997;15:3313-19.

23. Preusser P, Wilke H, Achterrath W, Fink U, Lenaz L, Heinicke A, et al. Phase II study with the combination etoposide, doxorubicin, and cisplatin in advanced measurable gastric cancer. J Clin Oncol 1989;7:1310-7.

24. Macdonald JS, Gohmann JJ. Chemotherapy of advanced gastric cancer: present status, future prospects. Semin Oncol 1988;15:429 (3 Suppl 4).

25. Ilson DH, Saltz L, Enzinger P, Huang Y, Kornblith A, Gollub M, et al. Phase II trial of weekly irinotecan plus cisplatin in advanced esophageal cancer. J Clin Oncol 1999;17:3270-5.

26. Ajani JA, Fodor M, Van Cutsem E, Tjulandin S, Moiseyenko V, Cabral F, et al. Multinational randomized phase II trial of docetaxel $(\mathrm{T})$ and cisplatin (C) with or without 5-fluorouracil (FU) in patients (pts) with advanced gastric or GE junction adenocarcinoma (AGC-AGEJC) (abstract). Proc Am Soc Clin Oncol 2000;19:957.

27. Chun HG, Puccio CA, Mittelman A. Weekly docetaxel and continuous infusion (CI) 5-fluorouracil (5-FU) in elderly patients (pts) with cancer of the stomach and distal esophagus (abstract 645). Proc Am Soc Clin Oncol 2001;20:162a.

28. Kettner E, Ridwelski K, Keilholz U, Gallkowski U, Gebauer $\mathrm{T}$, Kröning $\mathrm{H}$, et al. Docetaxel and cisplatin combination chemotherapy for advanced gastric cancer: results of two phase II studies (abstract 657). Proc Am Soc Clin Oncol 2001;20: 165 a.

29. Atiq OT, Kelsen DP, Shiu MH, Saltz L, Tong W, Niedzwiecki D, et al. Phase II trial of postoperative adjuvant intraperitoneal cisplatin and fluorouracil and systemic fluorouracil chemotherapy in patients with resected gastric cancer. J Clin Oncol 1993;11:42533.

30. Crookes P, Leichman CG, Leichman L, Tan M, Laine L, Stain S, et al. Systemic chemotherapy for gastric carcinoma followed by postoperative intraperitoneal therapy. Cancer 1997;79: $1767-75$. 